\title{
Performance Analysis of CoOperative Relaying IN NAKAGAMI-M FADING CHANNELS
}

\author{
B. Barua, Student Member, IEEE \\ and M. Z. I. Sarkar, Student Member, IEEE \\ School of Electronics and Information \\ Kyung Hee University, South Korea. \\ Email: \{b005barua,mzi_ruet@yahoo.com\}
}

\begin{abstract}
This paper is concerned with the analysis of exact symbol error probability (SEP) for cooperative diversity using amplify-and-forward $(A F)$ relaying over independent and non-identical Nakagami-m fading channels. The mathematical formulations for Probability Density Function (pdf) and Moment Generating Function (MGF) of a cooperative link have been derived for calculating symbol error probability with well-known MGF based approach taking M-ary Phase Shift Keying (MPSK) signals as input. The numerical results obtained from this research have been compared with different fading conditions. It is observed that the existence of the diversity link in a relay network plays a dominating role in error performance.
\end{abstract}

Keywords: Symbol Error Probability, Probability Density Function, Moment Generating Function, Nakagami-m fading.

\section{Introduction}

Dual hop cooperative relaying recently draws a considerable attention in wireless communication due to its low power requirement, large coverage and diversity [17]. The diversity provides robustness in system performance against variation in fading. Nonregenerative or Amplify-and-Forward (AF) relaying technique is the most promising one due to its less complexity. In this case, relay simply amplifies the received signals and retransmits towards the destination node. In particular, to scale the diversity order with the number of co-operative relays, multiple $\mathrm{AF}$ transmissions require orthogonalizing the relay channels.

Symbol error probability analysis is one of the crucial performance measures for any wireless systems. Various performance measures of relayed link have extensively done in many previous literatures. Pioneering works in [2-12] have analyzed error of relay networks from different perspectives. Outage and error Date of submission : 21.09.2008 Date of acceptance : 20.10.2010 analysis for blind relay has been proposed in [3-6] for Rayleigh and Nakagami-m fading channels. Lower bound on error is analyzed in [3] and [7] and upper bound in [4] and [9]. Asymptotic analysis of SEP has done in [10] and [11] especially for Rayleigh fading. And [12] has found the exact analysis of SEP for differential binary modulation over Nakagami$\mathrm{m}$ fading channels.

In this paper, we derive the exact symbol error probability of cooperative diversity of local CSI-assisted AF relaying in Nakagami-m fading. As per we know the exact SEP analysis for Nakagami-m in AF relay case for MPSK signaling has not done yet. We assumed the MRC combiner in the receiver using perfect weighting. We also draw different numerical results to distinguish the whole scenario of the AF relaying.

\section{System Model}

We consider a dual hop $\mathrm{K}$ single antenna, half duplex, cooperative relay network with single antenna source and destination, all operating in Amplify-and-Forward mode in Nakagami-m fading environment (fig. 1). Fading parameters are independent and non-identical. We also consider that all relays have its local (forward and backward channel) CSI only and use equal power in relay nodes irrespective its forward channel. We assume the direct link from source and destination also exists.

\section{Signal Model}

In the first hop, the source simply broadcast the message, and in second hop relays operate in orthogonal channels in time. In the receiver the signals are combined coherently using MRC, so receiver has perfect channel knowledge of all links. 
The signals received at the destination and relay nodes can be written as,

$$
\begin{aligned}
& y_{s d}=h_{s d} \sqrt{P_{s}} x+n_{s d} \\
& y_{1 i}=h_{1 i} \sqrt{P_{s}} x+n_{1 i} \\
& y_{2 i}=G_{i} h_{1 i} h_{2 i} \sqrt{P_{S}} x+G_{i} h_{2 i} n_{1 i}+n_{2 i}
\end{aligned}
$$

Where $x$ is the message signal with energy $P_{S}$, $y_{s d}$ is the received direct signal from the source. Other signals $y_{1 i}$ is the received signal at relay $i$ and $y_{2 i}$ is the received signal at the destination from relay $i . h_{s d}, h_{1 i}$ and $h_{2 i}$, are the complex fading amplitudes of direct link, $1^{\text {st }}$ hop and $2^{\text {nd }}$ hop of $i^{\text {th }}$ links respectively. The noise parameters $n_{s d}, n_{1 i}$ and $n_{2 i}$ are modeled as Zero Mean Circularly Symmetric Complex Gaussian (ZMCSCG) with variance $N_{s d}, N_{l i}$ and $N_{2 i}$ respectively.

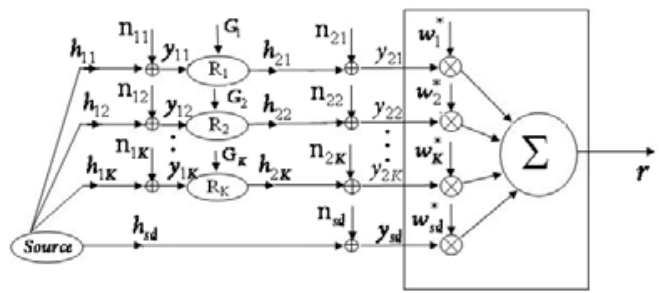

Fig. 1: System Model

The signal (2) is scaled by gain parameter $G_{i}$ such that energy from each node is constrained to $P_{R}$ such that, $P_{i} \leq P_{R}$. $P_{i}$ is the transmitting power of $i^{\text {th }}$ node.

The gain parameter of $i^{\text {th }}$ relay is given by,

$$
G_{i}=\sqrt{\frac{P_{i}}{\left(\left|h_{1 i}\right|^{2} P_{S}+N_{1 i}\right)}}
$$

The signal output of the combiner is,

$$
\begin{aligned}
r & =w_{s d}^{*} h_{s d} \sqrt{P_{S}} x+\sum_{i=1}^{K} w_{i}^{*} G_{i} h_{1 i} h_{2 i} \sqrt{P_{S}} x \\
& +\sum_{i=1}^{K}\left(w_{i}^{*} G_{i} h_{2 i} n_{1 i}+w_{i}^{*} n_{2 i}\right) \\
w_{s d}^{*}= & \frac{h_{s d}^{*}}{N_{s d}} \text { and } w_{i}^{*}=\frac{G_{i} h_{1 i}^{*} h_{2 i}^{*}}{G_{i}^{2}\left|h_{2 i}\right|^{2} N_{1 i}+N_{2 i}} \text { are }
\end{aligned}
$$

the weights for perfect coherency of direct link and relayed signal of $i^{\text {th }}$ relay respectively. The SNR at the output of the combiner can be described as the sum of two SNR's, one is direct link SNR and other is equivalent SNR of the relayed signals, say,

$\gamma=\gamma_{s d}+\gamma_{e q}$

Where, $\gamma_{e q}=\sum_{i=1}^{K} \frac{\gamma_{1 i} \gamma_{2 i}}{\gamma_{1 i}+\gamma_{2 i}+1}$

Let's

$\gamma_{i}=\frac{\gamma_{1 i} \gamma_{2 i}}{\gamma_{1 i}+\gamma_{2 i}+1}$

\section{Symbol Error Probability Analysis}

Considering Nakagami-m fading chan-nels, to find out the SEP we have to find out PDF, CDF and MGF of $\gamma_{i}$.

Let's random variable, $g_{k}=\frac{g_{1} g_{2}}{g_{1}+g_{2}+1}$,

where, $\gamma_{1}$ and $\gamma_{2}$ are Nakagami-m distributed random variables with pdf as,

$p_{\gamma}(\gamma)=\frac{m^{m} \gamma^{m-1}}{\bar{\gamma}^{m} \Gamma(m)} e^{-\left(\frac{m \gamma}{\bar{\gamma}}\right)} \quad \gamma \geq 0, m \geq \frac{1}{2}$,

where, $\bar{\gamma}$ is the average SNR. Then the CDF of $\gamma_{i}$ can be written as (8), shown at the bottom of the next page [7],

Where $K_{x}($.$) is the x^{\text {th }}$-order modified Bessel function of the second kind.

The PDF of $\gamma_{i}$ is given in (9), shown at the bottom of the next page,

Using this PDF the MGF can be calculated as,

$$
\begin{aligned}
\Phi_{\gamma_{k}}(s) & =E\left\{e^{-s \gamma}\right\} \\
& =\int_{0}^{\infty} e^{-s \gamma} p_{\gamma_{k}}(\gamma) d \gamma
\end{aligned}
$$

The solution of eq. (10) is not so simple. We can solve it numerically.

The exact expression of SEP can be written in well-known MGF based approach for MPSK signaling as, 


$$
\begin{array}{r}
P_{s}(e)=\frac{1}{\pi} \int_{0}^{\pi-\frac{\pi}{M}} \Phi_{\gamma}\left(\frac{\operatorname{Sin}^{2} \frac{\pi}{M}}{\operatorname{Sin}^{2} \theta}\right) d \theta \\
=\frac{1}{\pi} \int_{0}^{\pi-\frac{\pi}{M}}\left(1+\frac{\sin ^{2}(\pi / M) \bar{\gamma}_{s d}}{m_{s d} \sin ^{2} \theta}\right)^{-m_{s d}} \\
\times \Phi_{\gamma_{e q}}\left(\frac{\sin ^{2}(\pi / M)}{\sin ^{2} \theta}\right) d \theta
\end{array}
$$

\section{Numerical Results}

Numerical results of this paper are based on equal power allocation in all relay nodes. Power of all relay nodes are taken as $P_{t} / 2 K$, and $P_{s}=P_{t} / 2$. Where $P_{t}$ indicates the total power constrained of the system. The noise variances of all hops are taken as unity.

Figures 2, 3 and 4 show the symbol error probability as a function of SNR for different values of fading parameter in Nakagami-m fading channel with two $(K=2)$, five $(K=5)$ and ten $(K=10)$ relays respectively. The subscripts 0,1 and 2 in the fading parameter $m$ denote the direct link, first hop and second hop fading respectively. From these figures one can see that symbol error probability of a channel is highly affected by the fading and decreases with the decrease in severity of fading (as $\mathrm{m}$ goes from 1 to $\infty$ ).

$$
\begin{aligned}
& F_{\gamma_{k}}(\gamma)=1-\frac{2 m_{2}^{m_{2}}\left(m_{1}-1\right) ! e^{-\left(\frac{m_{1} \gamma}{\bar{\gamma}_{1}}+\frac{m_{2} \gamma}{\bar{\gamma}_{2}}\right)}}{{\overline{\gamma_{2}}}^{m_{2}} \Gamma\left(m_{1}\right) \Gamma\left(m_{2}\right)} \sum_{q=0}^{m_{1}-1} \sum_{l=0}^{q} \sum_{r=0}^{m_{2}-1}\left\{\frac{1}{q !}\left(\begin{array}{c}
q \\
l
\end{array}\right)\left(\begin{array}{c}
m_{2}-1 \\
r
\end{array}\right)\left(\frac{m_{1}}{\overline{\gamma_{1}}}\right)^{\frac{(2 q-l+r+1)}{2}}\left(\frac{m_{2}}{\overline{\gamma_{2}}}\right)^{\frac{(l-r-1)}{2}}\right. \\
& \left.\times \gamma^{\frac{\left(2 q+2 m_{2}-l+r+1\right)}{2}}(\gamma+1)^{\frac{(l+r+1)}{2}} K_{l-r-1}\left(2 \sqrt{\frac{m_{1} m_{2} \gamma(\gamma+1)}{\overline{\gamma_{1}} \overline{\gamma_{2}}}}\right)\right\} \\
& p_{\gamma_{k}}(\gamma)=\frac{-1}{\Gamma\left(m_{1}\right) \Gamma\left(m_{2}\right)}\left\{2 \overline { \gamma } _ { 2 } ^ { - m _ { 2 } } e ^ { - ( \frac { m _ { 1 } \gamma } { \overline { \gamma } _ { 1 } } + \frac { m _ { 2 } \gamma } { \overline { \gamma } _ { 2 } } ) } m _ { 2 } ^ { m _ { 2 } } ( m _ { 1 } - 1 ) ! \sum _ { q = 0 } ^ { m _ { 1 } - 1 } \sum _ { l = 0 } ^ { q } \sum _ { r = 0 } ^ { m _ { 2 } - 1 } \left\{\frac { 1 } { 2 q ! } \left(\left(\begin{array}{l}
q \\
l
\end{array}\right)\left(\begin{array}{c}
m_{2}-1 \\
r
\end{array}\right)\left(\frac{m_{1}}{\overline{\gamma_{1}}}\right)^{\frac{(2 q-l+r+1)}{2}}\right.\right.\right. \\
& \left.\times\left(\frac{m_{2}}{\bar{\gamma}_{2}}\right)^{\frac{(l-r-1)}{2}}\left(2 q-l-2 m_{2}-r-1\right) \gamma^{\frac{\left(2 q-l+2 m_{2}-r-1\right)}{2}-1}(\gamma+1)^{\frac{(l+r+1)}{2}} K_{l-r-1}\left(2 \sqrt{\frac{m_{1} m_{2} \gamma(\gamma+1)}{\bar{\gamma}_{1} \bar{\gamma}_{2}}}\right)\right) \\
& +\frac{1}{2 q !}\left(\left(\begin{array}{c}
q \\
l
\end{array}\right)\left(\begin{array}{c}
m_{2}-1 \\
r
\end{array}\right)\left(\frac{m_{1}}{\bar{\gamma}_{1}}\right)^{\frac{(2 q-l+r+1)}{2}}\left(\frac{m_{2}}{\bar{\gamma}_{2}}\right)^{\frac{(l-r-1)}{2}}(l+r+1) \gamma^{\frac{\left(2 q-l+2 m_{2}-r-1\right)}{2}}(\gamma+1)^{\frac{(l+r+1)}{2}-1}\right. \\
& \left.\times K_{l-r-1}\left(2 \sqrt{\frac{m_{1} m_{2} \gamma(\gamma+1)}{\bar{\gamma}_{1} \bar{\gamma}_{2}}}\right)\right)+\frac{1}{2 \sqrt{\frac{m_{1} m_{2} \gamma(\gamma+1)}{\bar{\gamma}_{1} \bar{\gamma}_{2}}} q !}\left(\left(\begin{array}{l}
q \\
l
\end{array}\right)\left(\begin{array}{c}
m_{2}-1 \\
r
\end{array}\right)\left(\frac{m_{1}}{\overline{\gamma_{1}}}\right)^{\frac{(2 q-l+r+1)}{2}}\left(\frac{m_{2}}{\bar{\gamma}_{2}}\right)^{\frac{(l-r-1)}{2}}\right. \\
& \times \gamma^{\frac{\left(2 q-l+2 m_{2}-r-1\right)}{2}}(\gamma+1)^{\frac{(l+r+1)}{2}}\left(\frac{m_{1} m_{2} \gamma}{\bar{\gamma}_{1} \bar{\gamma}_{2}}+\frac{m_{1} m_{2}(\gamma+1)}{\bar{\gamma}_{1} \bar{\gamma}_{2}}\right)\left(-K_{l-r-2}\left(2 \sqrt{\frac{m_{1} m_{2} \gamma(\gamma+1)}{\bar{\gamma}_{1} \bar{\gamma}_{2}}}\right)\right) \\
& \left.\left.\left.\left.-K_{l-r}\left(2 \sqrt{\frac{m_{1} m_{2} \gamma(\gamma+1)}{\overline{\gamma_{1}} \bar{\gamma}_{2}}}\right)\right)\right)\right\}\right\}-\frac{1}{\Gamma\left(m_{1}\right) \Gamma\left(m_{2}\right)}\left\{2 \bar{\gamma}_{2}^{-m_{2}} e^{-\left(\frac{m_{1} \gamma}{\bar{\gamma}_{1}}+\frac{m_{2} \gamma}{\bar{\gamma}_{2}}\right)} m_{2}^{m_{2}}\left(m_{1}-1\right) !\left(-\frac{m_{1}}{\overline{\gamma_{1}}}-\frac{m_{2}}{\overline{\gamma_{2}}}\right)\right. \\
& \times \sum_{q=0}^{m_{1}-1} \sum_{l=0}^{q} \sum_{r=0}^{m_{2}-1}\left\{\frac{1}{q !}\left(\begin{array}{l}
q \\
l
\end{array}\right)\left(\begin{array}{c}
m_{2}-1 \\
r
\end{array}\right)\left(\frac{m_{1}}{\overline{\gamma_{1}}}\right)^{\frac{(2 q-l+r+1)}{2}}\left(\frac{m_{2}}{\overline{\gamma_{2}}}\right)^{\frac{(l-r-1)}{2}} \gamma^{\frac{\left(2 q+2 m_{2}-l+r+1\right)}{2}}(\gamma+1)^{\frac{(l+r+1)}{2}}\right. \\
& \left.\left.\times K_{l-r-1}\left(2 \sqrt{\frac{m_{1} m_{2} \gamma(\gamma+1)}{\bar{\gamma}_{1} \bar{\gamma}_{2}}}\right)\right\}\right\}
\end{aligned}
$$




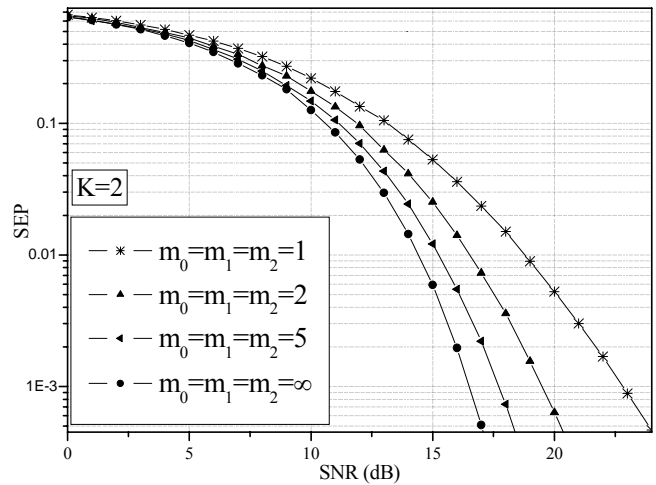

Fig.2: Symbol error probability versus SNR for different Nakagami-m parameter with $\mathrm{K}=2$.

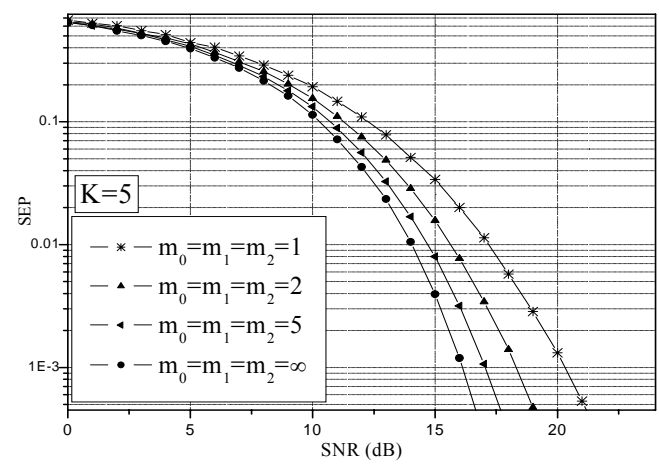

Fig.3: Symbol error probability versus SNR for different Nakagami-m parameter with $\mathrm{K}=5$.

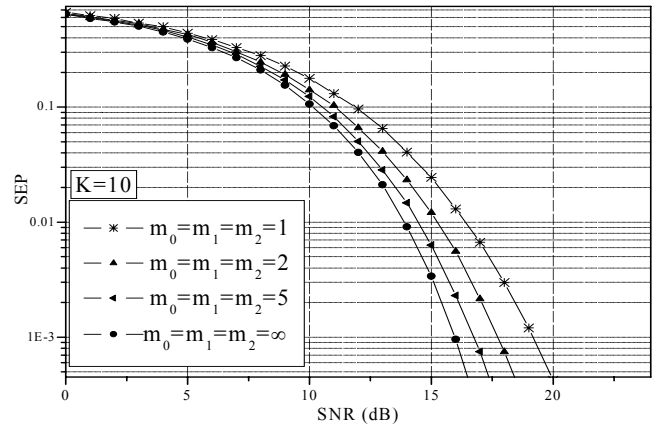

Fig.4: Symbol error probability versus SNR for different Nakagami-m parameter with $\mathrm{K}=10$

We also observe that the SEP performance is found to be dependent on the number of relays. As the number of relays increases, the SEP performance of a channel improves due to the improvement in diversity. The change of SEP performance in Rayleigh fading $(\mathrm{m}=1)$ channel with number of relays is more significant. So, one can improve the SEP of Rayleigh fading channel or any channel with considerable fading by changing the number of relays in the system. In case of AWGN channel $(m=\infty)$ the effect of number of relays on the SEP is not so much significant as we see in the Rayleigh fading case.

The effects of different fading in a particular relay link (i.e. Considering source-relaydestination as a link) have been described in Figs. 5, 6 and 7. In case of two relays as shown in Fig. 5, we see that in the direct link $\left(\mathrm{m}_{0}\right)$ the SEP performance of the system is highly affected by the fading (change of $\mathrm{m}_{0}$ for 1 to 5 ) of the channel. For relayed link, the effect fading on the SEP performance for second hop is higher than that the first hop which is illustrated in the figure by changing $\mathrm{m}_{1}$ and $\mathrm{m}_{2}$ from 1,5 to 5,1 . The SEP performance in the direct link as well as in the relayed link is affected by the number of relay which can be observed by comparing figures 5, 6 and 7 .

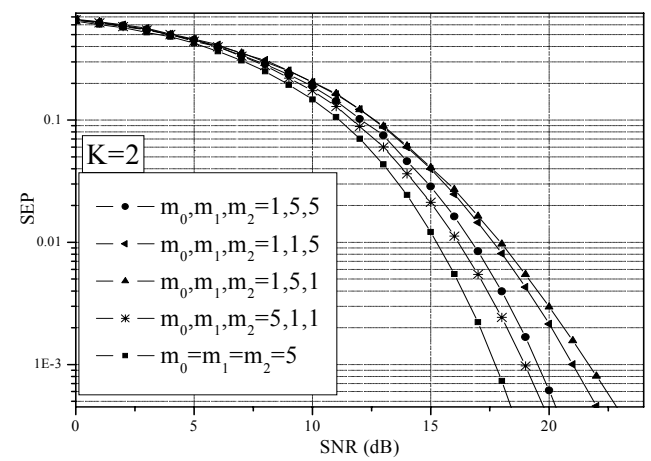

Fig.5: Symbol error probability versus SNR for different Nakagami-m parameter in the particular link with $\mathrm{K}=2$.

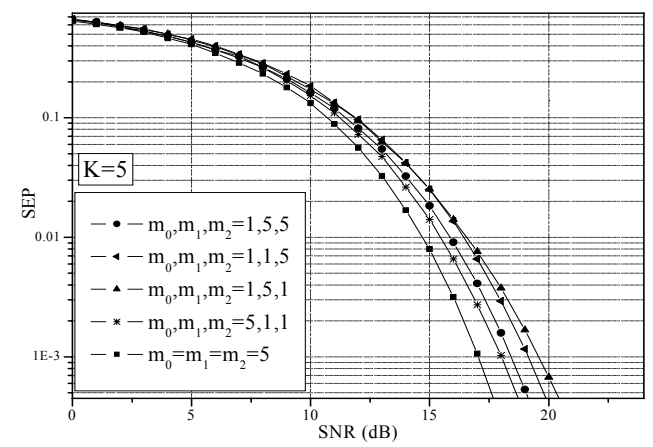

Fig.6: Symbol error probability versus SNR for different Nakagami-m parameter in the particular link with $\mathrm{K}=5$. 


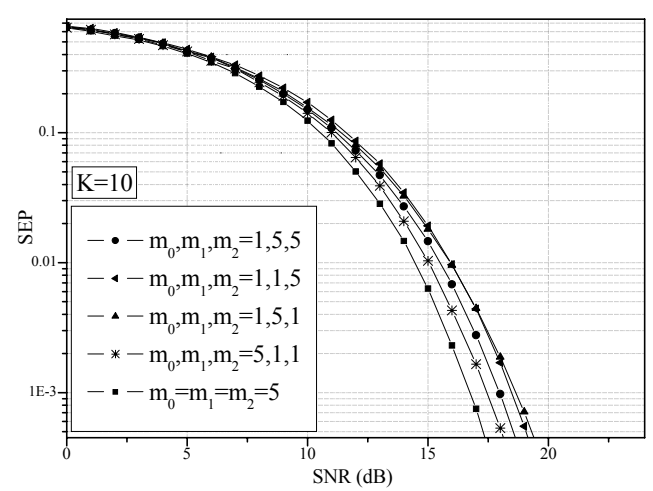

Fig.7: Symbol error probability versus SNR for different Nakagami-m parameter in the particular link with $\mathrm{K}=10$.

\section{Conclusion}

The error performance of a half-duplex AF relay network in Nakagami-m fading has been analyzed. The expression of pdf of received SNR is derived and then using well-known MGF based approach, the SEP of the network is calculated. Simulation results agree that higher values of $m$ provide less probability of error. We have also observed that the existence of direct link in different hops plays an important role for reducing error probability. Comparison of SEP in different fading conditions is done simply by varying the Nakagami-m parameter.

\section{References}

[1] J. N. Laneman, D. N. C. Tse, and G. W. Worn-ell, "Cooperative diversity in wireless networks: efficient protocols and outage behavior," IEEE Trans. Inf. Theory, vol. 50, December 2004, pp. 3062-3080.

[2] J. N. Laneman and G. W. Wornell, "Energy efficient antenna shar-ing and relaying for wireless networks," in Proc. IEEE Wireless Communications Networking Conf., Chicago, IL, October 2000, pp. 7-12.

[3] M. O. Hasna and M.-S. Alouini, "A performance study of dual-hop transmissions with fixed gain relays," IEEE Trans. Wireless Comm. vol. 3, November 2004, pp. 1963-1968.
[4] Yan Li and Shalinee Kishore "Asymptotic Analysis of Amplify-and-Forward Relaying in Nakagami-Fading Environments" IEEE Transactions on Wireless Communications, Vol. 6, No. 12, December 2007, pp. 4256-4262.

[5] Himal A. Suraweera, and George K. Karagiannidis, "Closed-Form Error Analysis of the Non-Identical Nakagami-m Relay Fading Channel" IEEE Communications Letters, Vol. 12, No. 4, April 2008, pp. 259-261.

[6] T. A. Tsiftsis, G. K. Karagiannidis, P. T. Mathiopoulos, and S. A. Kotsopoulos, "Nonregenerative dual-hop cooperative links with selection diversity," EURASIP J. Wireless Comm. Networking, vol.2006, article ID 17862, 2006, pages 1-8

[7] Ikki, S. Ahmed, M.H. "Performance Analysis of Cooperative Diversity Wireless Networks over Nakagami-m Fading Channel", IEEE Communications Letters, vol. 11, No. 4, April 2007, pp. 334-336.

[8] T. A. Tsiftsis, G. K. Karagiannidis, S.A. Kotsopoulos and F.-N. Pavlidou "BER analysis of collaborative dual-hop wireless transmissions" Electronics Letters, vol. 40, Issue. 11, 27 May 2004, pp. 679- 681.

[9] DimiIris A., George K. Karagiannidisf, Nikos C. Sagiasz, Theod-oros A. Tsiftsis, P. Takis Mathiopoulost and Stavros A. Kotsopoulos, "Dual hop wireless communications over nakagami fading" $59^{\text {th }}$ IEEE Vehicular Technology Conference, 17-19 Milan, Italy May 2004, pp. 2200-2204

[10] Y.Zhao, R. Adve, and T. J. Lim, .Symbol error rate of selection amplify-and-forward relay systems, IEEE Communications Letters, vol. 10, no. 11, November 2006, pp. 757.759.

[11] Paul A. Anghel and Mostafa Kaveh, "Exact Symbol Error Probab-ility of a Co-operative Network in a Rayleigh Fading Environment" IEEE Transactions on Wireless Communications, Vol. 3, No. 5, September 2004, pp. 1416-1421.

[12] Qiang Zhao and Hongbin Li "Performance Analysis of an Amplify-Based Differential Modulation for WireIess Relay Networks under Nakagami-m Fading Channels" IEEE 6th Workshop on Signal Processing Advances in Wireless Communications, 5-8 June 2005, pp. 211- 215. 